\title{
Influencia de la incorporación de la tomosíntesis en un centro de diagnóstico mamario
}

The impact of integrating breast tomosynthesis in a Breast Diagnostic Center Influência da incorporação da tomossíntese em um centro diagnóstico de mama

\author{
Gustavo Febles ${ }^{1}$, Cristina Balbiani², Graciela Vázquez²
}

\section{Resumen}

Introducción: se han producido múltiples avances tecnológicos en la historia de la mamografía. En los años más recientes surge la mamografía digital directa con tomosíntesis con mayores capacidades para detectar el cáncer.

Objetivo: evaluar el efecto de la nueva tecnología en el desempeño de la mamografía, en la Unidad de Diagnóstico Mamario del Hospital Británico.

Material y métodos: se compara el desempeño de la mamografía durante los años 2019-2020 en relación con los años 2010-2018. En el año 2019 se sustituyó la tecnología anterior por un mamógrafo digital directo con tomosíntesis, Hologic modelo Selenia Dimensions.

Resultados: en los años 2019 a 2020 se realizaron 10.725 mamografías. Se detectaron 84 cánceres y la tasa de detección de cáncer fue de 8/1.000. El VPP fue de 35\%. En los años 2010 a 2018 se realizaron 45.438 mamografías. Se detectaron 229 cánceres y la tasa de detección de cáncer fue de 5/1.000. EL VPP fue de 40\%. En relación a las manifestaciones imagenológicas, se destaca el aumento de las microcalcificaciones en el período 2019-2020, que pasaron de ser 7\% de los casos, a ser $19 \%$ de los casos. Las distorsiones aumentaron de $11 \%$ a 13\%. En relación a los tipos histológicos de cáncer, se destaca el porcentaje del carcinoma ductal in situ (CDIS) que fue 4 veces superior en el período 2019-2020 en relación al anterior (17\% de todos los casos detectados).

Conclusiones: con la incorporación de la tomosíntesis aumentó 60\% la tasa de detección de cáncer y el porcentaje de CDIS aumentó 4 veces.

Palabras clave: Tomosíntesis

Mamografía

Diagnóstico

Key words: $\quad$ Tomosynthesis

Mammography

Diagnosis

\footnotetext{
1. Médico Imagenólogo. Jefe de la Unidad De Diagnóstico Mamario del Hospital Británico.

2. Médico Imagenólogo. Integrante del staff de la Unidad De Diagnóstico Mamario del Hospital Británico.

Hospital Británico. Unidad De Diagnóstico Mamario (UDM).

Los autores declaran que no existen conflictos de interés.

Correspondencia: Dr. Gustavo Febles. Correo electrónico: febles.gustavo@gmail.com

Fecha recibido: 30/1/2021

Fecha aprobado: $28 / 7 / 2021$

Attribution-NonCommercial 4.0 International (CC BY-NC 4.0)
} 


\section{Introducción}

El cáncer de mama es el cáncer más frecuente en la mujer y la detección oportuna mediante la mamografía ha sido una estrategia de salud muy eficaz para reducir la mortalidad por esta enfermedad ${ }^{(1)}$.

Desde sus comienzos, se han producido múltiples avances tecnológicos en la mamografía: primero la mamografía analógica, luego la mamografía con digitalización indirecta (CR), luego la mamografía digital directa y en los años más recientes la mamografía digital directa con tomosíntesis.

Cada paso tecnológico implicó avances en la capacidad para detectar el cáncer, y son varios los parámetros que pueden analizarse para evaluar el rendimiento de la mamografía: tasa de detección de cáncer, tasa de interpretación anormal, valor predictivo positivo, características imagenológicas e histológicas de los cánceres diagnosticados ${ }^{(2)}$.

En la Unidad de Diagnóstico Mamario del Hospital Británico durante los años 2010 a 2012 se trabajó con mamografía analógica, en el año 2013 se digitalizaron las imágenes con un sistema de digitalización indirecta CR y finalmente en mayo de 2019 se sustituyó la tecnología anterior por un mamógrafo digital directo con tomosíntesis.

El objetivo de este trabajo es evaluar el efecto de la incorporación de la nueva tecnología en los parámetros que miden el desempeño de la mamografía, comparando los resultados del proceso diagnóstico durante el período 2019-2020, en relación al período 2010-2018.

\section{Material y métodos}

Se trata de un estudio retrospectivo en el que se compara el desempeño del centro en dos períodos diferentes: el período 2019 a 2020 y el período 2010 a 2018.

Durante el período 2010 a 2018 se trabajó con un mamógrafo Siemens modelo Mammomat Nova en forma analógica hasta el año 2013, y luego se incorporó un sistema de digitalización indirecta CR Fuji Profect.

Durante el período 2019 a 2020 se realizó un cambio de tecnología, sustituyéndose lo anterior por un mamógrafo digital directo con tomosíntesis Hologic modelo Selenia Dimensions.

En todos los casos y en ambos períodos analizados las mamografías fueron realizadas con las dos proyecciones básicas para cada mama (cráneo-caudal y oblicua-mediolateral). Se realizaron además las proyecciones mamográficas adicionales que fueron necesarias según cada caso en particular.

Cuando se incorporó la tomosíntesis, dicha técnica se utilizó en ambas proyecciones de cada mama y la imagen 2D se obtuvo mediante mamografía sintetizada
En ambos períodos la población estudiada incluyó pacientes asintomáticas que concurrieron por tamizaje, y pacientes con algún elemento clínico que concurrieron para diagnóstico.

En ambos períodos los mismos médicos radiólogos estuvieron trabajando en el análisis de las imágenes y elaboración de los informes.

La única modificación efectuada entre ambos períodos fue la incorporación de la nueva tecnología.

Las variables analizadas fueron las siguientes: número de mamografías realizadas, número de cánceres detectados, tasa de detección de cáncer (número de casos cada 1.000 mamografías realizadas), valor predictivo positivo, edad de presentación y las características imagenológicas e histológicas de los cánceres diagnosticados.

Para la realización de los cálculos se consideró como "patrón oro" para el diagnóstico de cáncer mamario el estudio histopatológico de la pieza quirúrgica.

Para el análisis estadístico se utilizó el software Epidat 3.1. Se utilizó un test de z para diferencia de proporciones y otro para diferencia de tasas de incidencia.

\section{Resultados}

\section{Número de mamografías realizadas, tasa de detección de cáncer y valor predictivo positivo}

Durante los años 2010 a 2018 se realizaron 45.438 mamografías. Se detectaron 229 cánceres y la tasa de detección de cáncer fue de 5/1.000.

El valor predictivo positivo (VPP), considerado como el porcentaje de cánceres diagnosticados en relación al total de mamografías positivas (BI-RADS 4 y 5 ), fue de $40 \%$.

Durante los años 2019 a 2020 se realizaron 10725 mamografías. Se detectaron 84 cánceres y la tasa de detección de cáncer fue de 8/1.000. El VPP fue de 35\%.

Entre ambos períodos se comprobó un aumento de $3 / 1.000(60 \%)$ en la tasa de detección de cáncer, mientras que el VPP disminuyó un 5\%.

La prueba de diferencias de proporciones y de tasas de incidencia indicó que las diferencias entre ambos períodos tenían validez estadística $(\mathrm{p}=0,0006$ en ambos casos, intervalo de confianza del 95\%).

Ya que recién se comenzó con la tomosíntesis en mayo de 2019, se compararon los datos de este año con los del año 2020, en el que se trabajó con tomosíntesis durante todo el año. Observamos que la tasa de detección de cáncer en 2019 fue de 7/1.000 y en 2020 fue de $9 / 1.000$. Se comprobó entonces un aumento de 2/1.000 $(29 \%)$ en dicha tasa en el 2020 en relación al año anterior. 
Tabla 1. Distribución de las manifestaciones imagenológicas de los cánceres diagnosticados.

\begin{tabular}{lcccc}
\hline & \multicolumn{2}{c}{ 2010-2018 } & & 2019-2020 \\
\cline { 2 - 5 } & $N^{0}$ & $\%$ & 50 & 60 \\
\hline Nódulo no circunscripto & 164 & 72 & 5 & 6 \\
Nódulo circunscripto & 13 & 10 & 11 & 13 \\
Distorsión & 22 & 7 & 2 & 19 \\
Microcalcificaciones & 17 & 3 & - & 2 \\
Asimetría focal & 8 & 0,4 & - & - \\
Conducto dilatado & 1 & 0,9 & - & - \\
Quiste complejo & 2 & 0,4 & - & - \\
Grupo de microquistes & 1 & 0,4 & & - \\
Lesión areolar (Paget) & 1 & & & \\
\hline
\end{tabular}

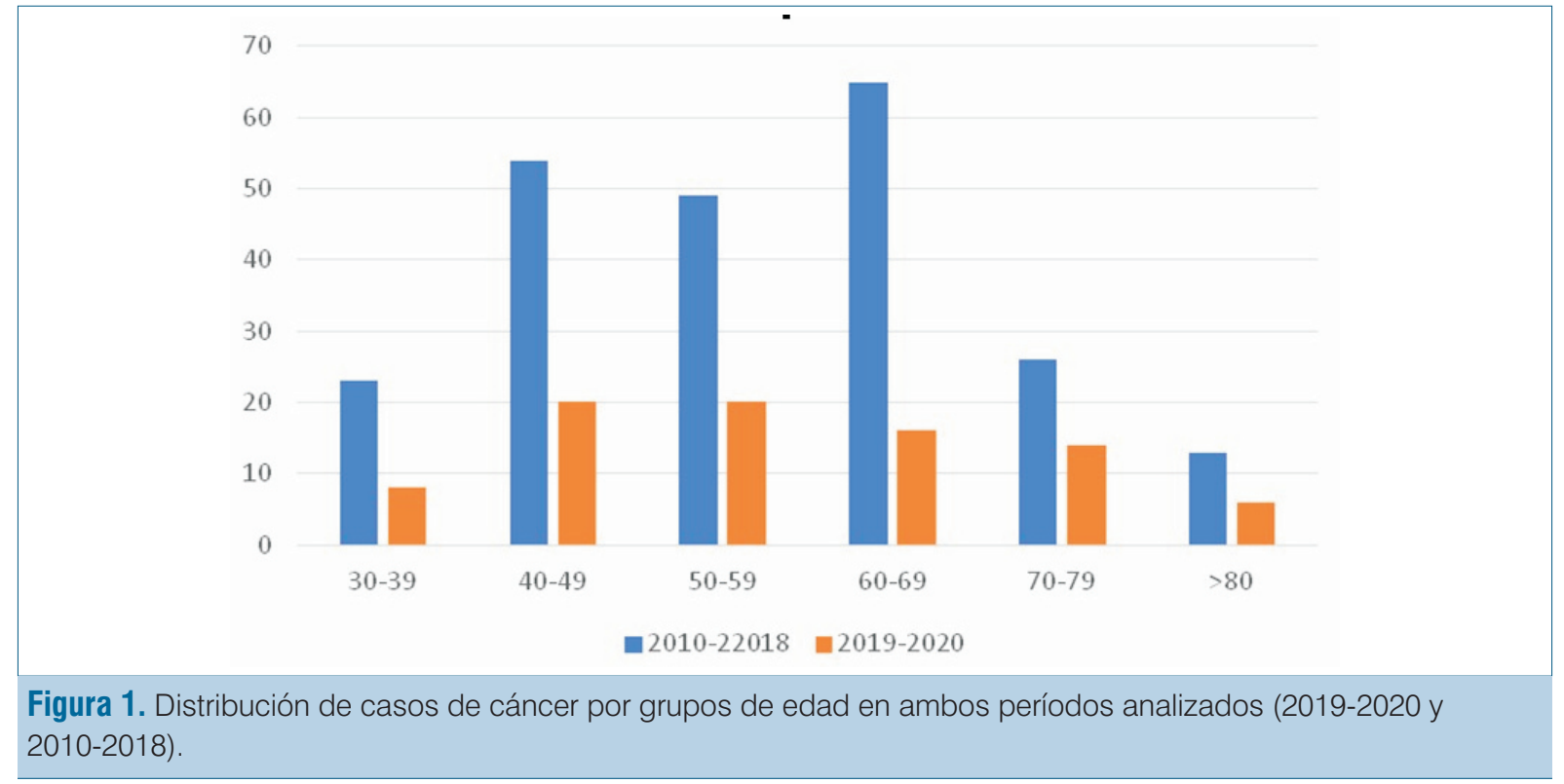

Al comparar el 2020 con el período 2010-2018, observamos que el aumento en la tasa de detección de cáncer fue de $4 / 1.000(80 \%)$.

\section{Distribución de los casos de cáncer según la edad de las pacientes}

En el período 2010-2018 el promedio de edad de las pacientes con cáncer mamario fue de 57 años (30-93), mientras que en el período 2019-2020 fue de 58 años (31-87).

En el primer período, 34\% de las pacientes con cáncer fue menor de 50 años, mientras que en el segundo período este valor fue de $33 \%$.
La distribución de casos de cáncer por grupos de edad se puede ver en la figura 1 .

Dicha distribución es bastante similar en ambos períodos, salvo por el pico de casos marcado en el grupo de 60 a 69 años en el período 2010-2018, que no es evidente en el siguiente período.

\section{Características imagenológicas de los cánceres diagnosticados.}

Las características imagenológicas de los cánceres diagnosticados se pueden ver en la tabla 1 .

En dicha tabla se observa un cambio notorio en la distribución de las diferentes manifestaciones imageno- 
Tabla 2. Distribución de los tipos histológicos de los cánceres diagnosticados.

\begin{tabular}{lcccc}
\hline & \multicolumn{2}{c}{$2010-2018$} & & \multicolumn{2}{c}{$2019-2020$} & \\
\cline { 2 - 5 } Histología & $N^{\circ}$ & $\%$ & 30 & $\%$ \\
\hline CDI & 131 & 58 & 27 & 36 \\
CDI/CDIS & 57 & 25 & 5 & 32 \\
CDI/CLI & 9 & 4 & 7 & 6 \\
CLI & 20 & 0,4 & 1 & 1 \\
CLI/CDIS & 1 & 4 & 14 & 17 \\
CDIS & 9 & & & 8 \\
\hline
\end{tabular}

CDI: carcinoma ductal invasivo; CDIS: carcinoma ductal in situ; CLI: carcinoma lobulillar invasivo.

lógicas entre ambos períodos. Se destaca el aumento proporcional de las microcalcificaciones, que pasaron de ser $7 \%$ de los casos en el primer período a ser $19 \%$ de los casos en el período siguiente. Las distorsiones aumentaron de $11 \%$ a $13 \%$.

\section{Características histológicas de los cánceres diagnosticados}

Las características histológicas de los cánceres detectados se pueden ver en la tabla 2.

En la distribución de los tipos histológicos de cáncer se destaca el porcentaje del carcinoma ductal in situ (CDIS), que es 4 veces superior en el período 2019-2020 en relación al anterior, ubicándose en $17 \%$ de todos los casos detectados.

El tamaño promedio de los cánceres invasores fue de $19 \mathrm{~mm}$ en el período $2010-2018$ y de $17 \mathrm{~mm}$ en el siguiente período.

En el período 2010-2018 71\% de los cánceres invasores no presentó metástasis en los ganglios axilares. Esto ocurrió en $73 \%$ de los cánceres invasores en el período 2019-2020.

\section{Discusión}

Las publicaciones internacionales son coincidentes en relación con el aumento en la tasa de detección de cáncer luego de la incorporación de la mamografía digital directa con tomosíntesis, aunque varía la magnitud de ese aumento ${ }^{(3)}$.

En comparación con la mamografía digital directa sola, la tomosíntesis con mamografía digital directa se asocia con aumentos en la tasa de detección de cáncer que van de 1,2 a 4,6 por 1.000 estudios realizados.

En nuestra serie la comparación se realizó entre el período en el cual se trabajó con mamografía digital directa y tomosíntesis (2019-2020), en relación al período en el cual se trabajó con mamografía analógica y luego con digitalización indirecta (2010-2018). Entre ambos períodos se comprobó un aumento de 3/1.000 (60\%) en la tasa de detección de cáncer. Sin embargo, al comparar el año en el que se trabajó exclusivamente con mamografía digital directa y tomosíntesis (2020) con el período 2010-2018, el aumento en la tasa de detección de cáncer fue de 4/1.000 (80\%).

Varios estudios han mostrado que la incorporación de la tomosíntesis lleva a un aumento en el valor predictivo positivo, definido como el porcentaje de estudios positivos en los cuales se confirma el diagnóstico de cáncer ${ }^{(4-11)}$. Sin embargo la evidencia no es consistente ${ }^{(12)}$.

En nuestra serie el valor predictivo positivo disminuyó en el período 2019-2020 en relación al período anterior, pasó de $40 \%$ a $35 \%$.

Varios estudios han demostrado que el aumento de los cánceres detectados con la tomosíntesis se debe principalmente a un aumento en la detección de cánceres invasivos en lugar de $\operatorname{CDIS}^{(4,6,7,12)}$.

En nuestra serie ocurrió lo contrario, el porcentaje de CDIS fue 4 veces superior en el período 2019-2020 en relación al período anterior. Es probable que esto se deba a que no estamos comparando el desempeño de la tomosíntesis en relación a la mamografía digital directa sola, sino que la comparación se hace en relación a mamografía analógica y mamografía con digitalización indirecta.

Con respecto a las manifestaciones imagenológicas de los cánceres diagnosticados, los estudios indican que la tomosíntesis aumenta la detección de distorsiones arquitecturales $^{(13,14)}$. En nuestra serie la detección de distorsiones arquitecturales aumentó un $3 \%$ en el período 2019-2020 en relación al período anterior.

El gran aumento en el diagnóstico de CDIS en nuestra serie explica el aumento en la detección de microcalcificaciones. Esto se debe a que la manifestación image- 
nológica más frecuente de dicha patología son las microcalcificaciones.

Como limitación de nuestro trabajo debemos señalar que no se discriminó entre las pacientes asintomáticas que concurrieron por tamizaje de las pacientes con elementos clínicos que concurrieron para diagnóstico. Tampoco fue posible calcular la tasa de detección anormal (tasa de rellamada en el caso del tamizaje) ya que, al brindarse una atención presencial en todos los casos, no se utiliza la categoría 0 del BI-RADS y entonces se hacen los procedimientos adicionales que sean necesarios en el momento de la consulta inicial.

\section{Conclusiones}

El estudio compara el desempeño de la Unidad de Diagnóstico Mamario del Hospital Británico en dos períodos diferentes: 2019-2020 y 2010-2018.

El único cambio efectuado entre ambos períodos fue la incorporación de la nueva tecnología en el año 2019, consideramos que este cambio fue el causante de un aumento de 3/1.000 (60\%) en la tasa de detección de cáncer. También se produjo un aumento en la detección de microcalcificaciones como manifestación de cáncer, que pasó de 7\% a 19\% de los casos y un aumento de las distorsiones arquitecturales como manifestación de cáncer, que pasaron de $11 \%$ a $13 \%$ de los casos.

En cuanto a la distribución de los tipos histológicos de cáncer, se destaca el porcentaje del CDIS que fue 4 veces superior en el período 2019-2020 en relación al anterior, ubicándose en $17 \%$ de todos los casos detectados.

\section{Agradecimientos}

Dra. Mariela Garau (Prof. Agregada de Métodos Cuantitativos. Facultad de Medicina, UDELAR).

\section{Abstract}

Introduction: significant technological progress has been made in the history of mammography. Recently, direct digital mammography plus tomosynthesis arrived and improved breast cancer screening.

Objective: to evaluate the effects of new technology in the performance of mammography, at the Breast Diagnostic Service of the British Hospital.

Method: mammography performance during 20192020 was compared to that between 2010 and 2018.

In 2019 the previous tehcnology was substituted by a direct digital mammogram with tomosynthesis, Hologic, Selenia Dimensions model.

Results: 10.725 mammographies were done in 2019-2020. Cancer was detected in 84 cases and the cancer detection rate was 8 per 1000 persons. PPV was $35 \%$.
45.438 mammographies were done in 2010-2018. Cancer was detected in 229 cases and the cancer detection rate was 5 per 1.000 persons. PPV was $40 \%$.

As to images, it is worth pointing out an increase in microcalcifications between 2019 and 2020, when they grew from $7 \%$ of cases to $19 \%$ of cases and distortions increased from $11 \%$ to $13 \%$.

DCIS was 4 times greater in 2019-2020 when compare to the previous period of time $(17 \%$ of all detected cases).

Conclusions: the integration of tomosynthesis increased $60 \%$ the cancer detection rate and the DCIS was 4 times greater.

\section{Resumo}

Introdução: a história da mamografia registra vários avanços tecnológicos. Nos anos mais recentes, a mamografia digital direta com tomossíntese surgiu com uma maior capacidade de detectar a presença de câncer. Em 2019 a Unidade de Diagnóstico de Mama (UDM) do Hospital Britânico no Uruguai, incorporou um mamógrafo digital direto com tomossíntese, Hologic modelo Selenia Dimensions.

Objetivo: avaliar desempenho do mamógrafo digital direto com tomossíntese na UDM.

Material e métodos: faz-se uma comparação dos resultados das mamografias durante dois períodos 2019-2020 e 2010-2018.

Resultados: no período 2019 a 2020 foram realizadas 10.725 mamografias; 84 cânceres foram detectados com una taxa de detecção de $8 / 1000$. O valor preditivo positivo (VPP) foi de $35 \%$. No período $2010-2018$, foram realizadas 45.438 mamografias, 229 cânceres foram detectados com una taxa de detecção de 5/1000. O VPP foi de $40 \%$.

Em relação às manifestações de imagem, destaca-se o aumento das microcalcificações no período 2019-2020, que passou de 7\% para 19\% dos casos. As distorções aumentaram de $11 \%$ para $13 \%$.

Em relação aos tipos histológicos de câncer, destaca-se a porcentagem de carcinoma ductal in situ (CDIS), que foi quatro vezes maior no período 2019-2020 do que no anterior ( $17 \%$ do total de casos detectados).

Conclusões: com a incorporação da tomossíntese, a taxa de detecção de câncer aumentou $60 \%$ e a porcentagem de CDIS aumentou quatro vezes.

\section{Bibliografía}

1. Febles G. Revisión de los beneficios del screening del cáncer de mama y análisis de las controversias. Rev Imagenol 2015; 19(1):09-18.

2. Febles G, Estellano F, Simón O. Auditoría de los resultados de la mamografía diagnóstica en el Centro de Diagnóstico 
Mamario de la Asociación Española. Rev Méd Urug 2009; 25(1):5-13.

3. Chong A, Weinstein S, McDonald E, Conant E. Digital breast tomosynthesis: concepts and clinical practice. Radiology 2019; 292(1):1-14.

4. Conant EF, Beaber EF, Sprague BL, Herschorn SD, Weaver DL, Onega T, et al. Breast cancer screening using tomosynthesis in combination with digital mammography compared to digital mammography alone: a cohort study within the PROSPR consortium. Breast Cancer Res Treat 2016; 156(1):109-16.

5. Skaane P, Bandos AI, Gullien R, Eben EB, Ekseth U, Haakenaasen $\mathbf{U}$, et al. Comparison of digital mammography alone and digital mammography plus tomosynthesis in a population-based screening program. Radiology 2013; 267(1): 47-56.

6. Ciatto S, Houssami N, Bernardi D, Caumo F, Pellegrini M, Brunelli S, et al. Integration of 3D digital mammography with tomosynthesis for population breast-cancer screening (STORM): a prospective comparison study. Lancet Oncol 2013; 14(7):583-9.

7. Caumo F, Zorzi M, Brunelli S, Romanucci G, Rella R, Cugola L, et al. Digital breast tomosynthesis with synthesized two-dimensional images versus full-field digital mammography for population screening: outcomes from the Verona screening program. Radiology 2018; 287(1):37-46.

8. Hofvind S, Hovda T, Holen AS, Lee CI, Albertsen J, Bjørndal H, et al. Digital breast tomosynthesis and synthetic 2D mammography versus digital mammography: evaluation in a population-based screening program. Radiology 2018; 287(3):787-94.

9. Friedewald SM, Rafferty EA, Rose SL, Durand MA, Plecha DM, Greenberg JS, et al. Breast cancer screening using tomosynthesis in combination with digital mammography. JAMA 2014; 311(24):2499-507.

10. Greenberg JS, Javitt MC, Katzen J, Michael S, Holland AE. Clinical performance metrics of 3D digital breast tomosynthesis compared with 2D digital mammography for breast cancer screening in community practice. AJR Am J Roentgenol 2014; 203(3):687-93.

11. Pattacini P, Nitrosi A, Giorgi Rossi P, Iotti V, Ginocchi V, Ravaioli S, et al. Digital mammography versus digital mammography plus tomosynthesis for breast cancer screening: the Reggio Emilia Tomosynthesis randomized trial. Radiology 2018; 288(2):375-85.

12. Giampietro RR, Gama MV, Molina SA, Weber SA, Dos Santos Nunes-Nogueira V. Accuracy and Effectiveness of Mammography versus Mammography and Tomosynthesis for Population-Based Breast Cancer Screening: A Systematic Review and Meta-Analysis. Sci Rep 2020; 10(1):7991. doi: 10.1038/s41598-020-64802-x.

13. Partyka L, Lourenco AP, Mainiero MB. Detection of mammographically occult architectural distortion on digital breast tomosynthesis screening: initial clinical experience. AJR Am J Roentgenol 2014; 203(1):216-22.

14. Dibble EH, Lourenco AP, Baird GL, Ward RC, Maynard AS, Mainiero MB. Comparison of digital mammography and digital breast tomosynthesis in the detection of architectural distortion. Eur Radiol 2018; 28(1):3-10.

\section{Contribución de autores}

Gustavo Febles, ORCID 0000-0002-4567-6472. Concepción, diseño, ejecución, análisis, interpretación de los resultados, redacción, revisión crítica.

Cristina Balbiani, ORCID 0000-0003-1706-4812. Ejecución, análisis, revisión crítica.

Graciela Vázquez, ORCID 0000-0001-9847-544X. Ejecución, análisis, revisión crítica. 